\title{
The Diagnostic Validity of TROP-2 in Recognizing Papillary Thyroid Carcinoma
}

\author{
NEHAL M. NABIL, M.Sc.; ASMAA G. ABDOU, M.D.; MOHAMMED I. SHABAN, M.D. and \\ RANIA A. ABDALLAH, M.D.
}

The Department of Pathology, Faculty of Medicine, Menoufia University, Menoufia, Egypt

\begin{abstract}
Background: Thyroid cancer is currently the third fastest rising cancer diagnosis in the United States and represents $80.3 \%$ of endocrine neoplasms and $2.6 \%$ of total malignancies in Egypt with male to female ratio is 1:2. Papillary thyroid carcinoma (PTC) represents the most common histologic type of thyroid carcinoma in Egypt representing 83.3\%. Diagnosis of papillary thyroid carcinoma is not always straight forward because some lesions exhibit equivocal nuclear features making the distinction of benign from malignant lesions is difficult. So it is necessary to use ancillary diagnostic tools to reach accurate diagnosis. TROP-2 is a trans-membranous glycoprotein, was originally identified in human trophoblast and choriocarcinoma cell lines and was subsequently reported to be over-expressed in a variety of human carcinomas. Few studies have verified the diagnostic power of TROP2 in PTC suggesting that it could be a potential novel immunohistochemical marker for identification of both classic and follicular variants of PTC
\end{abstract}

Aim of Study: The aim of this study is to review the diagnostic value of TROP-2 in papillary thyroid carcinoma (PTC) in English literature.

Material and Methods: From Medline databases (PubMed, Medscape and Google scholar) and also materials available on the Internet from 2014 to 2017.

The initial search presented 5 articles that studied the diagnostic role of TROP-2 in differentiating PTC from non malignant thyroid lesions and were performed on histological specimens only (cytological specimens were excluded).

If the studies did not fulfill the inclusion criteria, they were excluded. Study quality assessment included whether ethical approval was gained, eligibility criteria specified, appropriate controls, and adequate information and defined assessment measures.

A structured systematic review was performed with the results tabulated.

Results: TROP-2 sensitivity in total papillary thyroid carcinoma cases ranged from $50 \%$ to $86.8 \%$ passing through

Correspondence to: Dr. Nehal M. Nabil, The Department of Pathology, Faculty of Medicine, Menoufia University, Menoufia, Egypt
$58.3 \%$ to $81.5 \%$ and $81.6 \%$. Regarding TROP-2 specificity it was $100 \%$ in 4 out of 5 articles and $89.3 \%$ in the remaining one. Four articles showed $100 \%$ of positive predictive value (PPV) and one article demonstrated $84.6 \%$. The negative predicitive value (NPV) ranged from $74.1 \%$ to $92.4 \%$ and the diagnostic accuracy ranged from $79.4 \%$ to $94.3 \%$. As regard non follicular variant PTC (non FVPTC) TROP-2 sensitivities ranged between $81.5 \%$ and $98.7 \%$. The NPV ranged between $84 \%$ and $99.6 \%$, while diagnostic accuracy ranged between $88.6 \%$ and $99.7 \%$. The PPV was $100 \%$ in four articles and $82.4 \%$ in one article.

Conclusion: TROP-2 could be considered as a reliable immunostaining marker in diagnosing papillary thyroid carcinoma especially non follicular variant owing to its high specificity and sensitivity. TROP-2 is a specific rather than sensitive as its specificities in 4 studies revealed $100 \%$. Therefor it is expected to be negative in benign and non-neoplastic thyroid lesions reducing the overdiagnosis of PTC and discriminating it from many mimickers.

Key Words: TROP-2 antigen - Immunohistochemistry Papillary-Thyroid cancer.

\section{Introduction}

THYROID cancer is currently the third fastest rising cancer diagnosis in the United States. Thyroid cancer represents $3.4 \%$ of all new cancer cases in the U.S.A bout 56,870 new cases of thyroid cancer has been reported in United states in 2017 according to SEER Stat Fact Sheet, 2017 [1].

Thyroid carcinoma represents $80.3 \%$ of endocrine neoplasms and $2.6 \%$ of total malignancies in Egypt. The male to female ratio is $1: 2$ and the age of patients ranges between 14 and 90 with a mean of 41 years. Papillary thyroid carcinoma (PTC) represents the most common histologic type of thyroid carcinoma in Egypt representing 83.3\% followed by follicular carcinoma (8.4\%) [2] .

The diagnostic criteria for PTC have been established for more than 50 years ago [3], pathologic 
examination through routine hematoxylin and eosin staining is the 'gold standard' for diagnosis of PTC [4]. Although the initial diagnostic criterion for the diagnosis of papillary thyroid carcinoma was the presence of papillary growth pattern, nuclear criteria for the diagnosis are very important as well because of emerging of different growth patterns lacking papillary architecture. Therefore, in the past few decades, the nuclear features have become the diagnostic hallmark of the tumor and growth pattern became of lesser importance [5]

Diagnosis of papillary thyroid carcinoma is not always straight forward because some lesions exhibit equivocal nuclear features making the distinction of benign from malignant lesions is difficult. Furthermore, papillary and follicular architectures together with nuclear irregularity may be seen in both benign and malignant lesions [6-8]

Moreover, severe chronic lymphocytic thyroiditis, Hashimoto's thyroiditis, and reactive atypia result in nuclear morphology similar to that of papillary carcinoma, with nuclear enlargement, chromatin clearing, and even grooving [9]. In contrast, follicular variant of PTC and other variants may cause, if the nuclear features of PTC are insufficiently determined, difficult problems in differentiation from follicular thyroid carcinoma (FTC), follicular adenoma (FA), or even from dominant nodule of multi-nodular goiter [10]

Taking in consideration that over-or underdiagnosis of PTC have a great impact on patient prognosis, it is necessary to use ancillary diagnostic tools to discriminate these morphologically overlapping diseases using effective markers carrying high sensitivity and specificity [11].

The tumor-associated calcium signal transducer (TACSTD) gene family is comprised of two closely related genes, TACSTD1 and TACSTD2, encoding epithelial cell adhesion molecule trophoblastic cell surface antigen (TROP-1) and (TROP-2), respectively $[\mathbf{1 2 , 1 3}$. TROP-2 is a transmembranous glycoprotein, was originally identified in human trophoblast and choriocarcinoma cell lines [14-16] TROP-2 was subsequently reported to be overexpressed in a variety of human carcinomas, including colorectal, gastric and pancreatic carcinomas, squamous cell carcinoma of the oral cavity, non-small cell carcinoma of the lung, endometrial and ovarian malignancies, and only rarely in normal tissues [16-18]. TROP-2 over-expression in human carcinomas is associated with tumor aggressiveness and poor prognosis $[\mathbf{1 9 , 2 0 ]}$. In recent years, TROP-
2 has been actively studied as a prognostic marker and an attractive immunotherapeutic target in human cancer treatment [21,22].

Few studies have verified the diagnostic power of TROP2 in PTC suggesting that it could be a potential novel immunohistochemical marker for identification of both classic and follicular variants of PTC. Furthermore, those studies have also demonstrated that TROP2 has become more specific than other traditional markers (CK19, HBME-1, and galectin 3) $[\mathbf{2 3 , 2 4 ]}$

The aim of this study is to review the diagnostic value of TROP-2 in PTC in English literature.

\section{Patients and Methods}

\section{Search strategy:}

We reviewed papers in the electronic databases from 2014 to 2017 that discussed the diagnostic role of TROP-2 on papillary thyroid carcinomas using medline databases (PubMed, Medscape and Google scholar) and also materials available on the Internet. We used TROP-2, thyroid cancer, papillary thyroid carcinoma and immunohistochemistry of thyroid carcinoma searching terms. The search was performed.

\section{Study selection:}

All the studies were assessed independently for inclusion. They were included if they fulfilled the following criteria.

- Published in the English language.

- Published in peer reviewed journals.

- Focused on TROP-2 and thyroid neoplasms.

- Discussed the potential diagnostic utility of TROP-2 on thyroid carcinomas.

- Done on histological specimens of papillary thyroid carcinoma.

\section{Data extraction:}

If the studies did not fulfill the above criteria, they were excluded such as TROP-2 in non papillary thyroid carcinomas as follicular, anaplastic and medullary types, overexpression of TROP-2 in carcinomas other than those of thyroid origin and lastly TROP-2 studies on PTC cytological samples.

Data from each eligible study were independently abstracted in duplicate using a data collection form to capture information on study characteristics, interventions, quantitative results reported for each outcome of interest. Conclusions and comments on each study were made. 
Due to small number of the studied articles and heterogeneity in the collected data, It was not possible to perform meta-analysis. Significant data were collected. Thus, a structured review was performed with the result tabulated.

The analyzed publications were evaluated according to evidence-based medicine (EBM) criteria using the classification of the U.S. Preventive Services Task Force \& UK National Health Service protocol for EBM in addition to the Evidence Pyramid.

\section{U.S. Preventive Services Task Force:}

- Level I: Evidence obtained from at least one properly designed randomized controlled trial.

- Level II-1: Evidence obtained from welldesigned controlled trials without randomization.

- Level II-2: Evidence obtained from welldesigned cohortor case-controlanalytic studies, preferably from more than one center or research group.

- Level II-3: Evidence obtained from multiple time series with or without the intervention. Dramatic results in uncontrolled trials might also be regarded as this type of evidence.

- Level III: Opinions of respected authorities, based on clinical experience, descriptive studies, or reports of expert committees.

\section{Quality assessment:}

The quality of all the studies was assessed. Important factors included, study design, attainment of ethical approval, evidence of a power calculation, specified eligibility criteria, appropriate controls, adequate information, and specified assessment measures. It was expected that confounding factors would be reported and controlled for and appropriate data analysis carried out in addition to an explanation of missing data.

\section{Data synthesis:}

A structured systematic review was performed with the results tabulated. Calculation of TROP2's sensitivity, specificity, positive predictive value (PPV), negative predictive value (NPV) and diagnostic accuracy (DA) were done.

\section{Results}

\section{Study selection and characteristics:}

In total, 5 potentially relevant publications were identified. All of these articles were included in the review as they were deemed eligible by fulfilling the inclusion criteria. The 5 articles included in this review were human, case control or cohort studies using immunohistochemical staining on paraffin blocks of PTC cases. Data were obtained from PubMed, Medscape and Google scholar. Comparisons were made by structured review with the results tabulated. Calculation of the sensitivity, specificity, PPV, NPV and diagnostic accuracy of total PTC cases in addition to non follicular variant of PTC and follicular variant PTC in the collected articles regarding TROP-2 were performed.

The studies were analyzed with respect to the study design using the classification of the U.S. Preventive Services Task Force \& UK National Health Service protocol for EBM.

\section{Types of specimens:}

We collected data from the 5 relevant articles that studied the diagnostic role of TROP-2 in papillary thyroid carcinoma on histological specimens. The investigated malignant cases in those articles included papillary thyroid carcinoma and its variants (classic variant, tall cell variant and microcarcinoma which are totally included under non follicular category in addition to follicular variant), follicular thyroid carcinoma, medullary thyroid carcinoma, poorly differentiated and anaplastic carcinoma. On the other hand the non malignant cases comprised wide range of different thyroid lesions including non-neoplastic cases such as Hashimoto thyroiditis, Grave's disease and multinodular goitre, benign neoplasm as follicular adenoma together with normal thyroid tissue (Table 1).

Diagnostic value of TROP-2 in recognizing papillary thyroid carcinoma (Table 2).

\section{I- Total papillary cases:}

Data collected from the selected 5 articles that investigated TROP-2 immunohistochemical staining on papillary thyroid carcinoma revealed that TROP-2 sensitivity ranged from $50 \%$ (21 out of 42 PTC cases were positive) to $86.8 \%$ (33 out of 38 PTC cases were positive) passing through $58.3 \%$ (63 out of 108 PTC cases were positive) to $81.5 \%$ (66 out of 81 PTC cases were positive) and lately $81.6 \%$ (93 out of 114 PTC cases were positive). Concerning TROP-2 specificity it was $100 \%$ in 4 out of 5 articles except for only one article [25] where the specificity was $89.3 \%$ (50 out of 56 cases were true negative and only six cases were false positive). Four articles showed 100\% PPV and one article [25] demonstrated $84.6 \%$ as PPV. The NPV ranged from $74.1 \%$ to $92.4 \%$ and the diagnostic accuracy ranged from $79.4 \%$ to $94.3 \%$ (Table 2). 

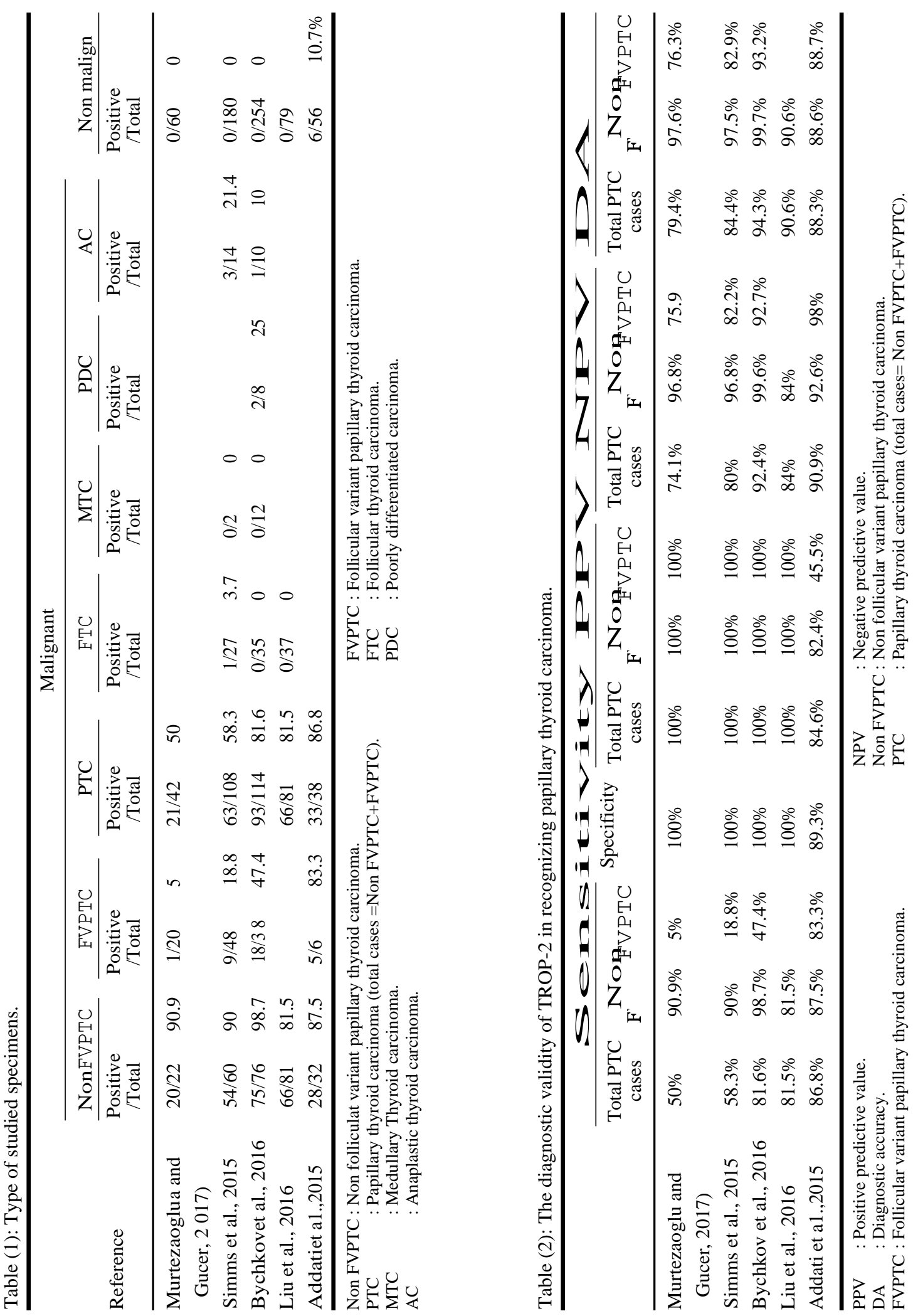


\section{II- Non follicular variant PTC (NFVPTC):}

Concerning the diagnostic validity of TROP2 in non follicular variant papillary thyroid carcinoma; The sensitivity ranged from 81.5 (66 out of 81 NFVPTC cases were positive) to $98.7 \%$ (75 out of 76 NFVPTC cases were positive) passing through 87.5 (28 out of 32 NFVPTC cases were positive), $90 \%$ (54 out of 60 NFVPTC cases were positive) and $90.9 \%$ (20 out of 22 NFVPTC cases were positive). The PPV was $100 \%$ in four articles and $82.4 \%$ in one article [25]. The NPV ranged from $84 \%$ to $99.6 \%$. TROP-2 diagnostic accuracy ranged from $88.6 \%$ to $99.7 \%$ (Table 2).

\section{III- Follicular variant PTC (FVPTC):}

Considering FVPTC, TROP-2 diagnostic validity showed a low sensitivity that ranged from $5 \%$ (1 out of 20 FVPTC cases was positive) to $83.3 \%$ ( 5 out of 6 FVPTC cases were positive) passing through $18.8 \%$ (9 out of 48FVPTC cases were positive) and $47.4 \%$ (18 out of 38 FVPTC cases were positive). The PPV was $100 \%$ in four articles and $45.5 \%$ in one article [25]. The NPV ranged from $75.9 \%$ to $98 \%$, while the diagnostic accuracy ranged from $76.3 \%$ to $93.2 \%$ (Table 2 ).

\section{Discussion}

Papillary thyroid carcinoma diagnosis is based on classic nuclear features as elongated nuclei with inconspicuous eccentric nucleoli and crinkled nuclear membranes, chromatin clearing, and intranuclear grooves and holes. Although a majority of papillary thyroid cancers can be diagnosed and classified on the basis of histopathologic criteria, there are a group of cases in which benign thyroid tissue or lesions can mimic nuclear cytologic features or the architecture and growth pattern of PTC, posing diagnostic problems [26,27]. Also thyroid neoplasms with follicular patterns as follicular variant of papillary thyroid carcinoma, follicular adenoma and follicular carcinoma often pose difficulties in accurate diagnosis during the assessment of cytologic and histologic specimens [28]. Thus we are in need of a new tool or marker with especially high sensitivity and specificity trying to solve these problems.

In the current review, the sensitivity of TROP2 in diagnosing papillary thyroid carcinoma ranged from $50 \%$ [29] to $86.6 \%$ [25]. Reduced sensitivity of TROP-2 to 50\% according to (Murtezaoglu and Gucer, 2017) [29] may be explained by absence of its expression in most of follicular variant of PTCs compared to its intense and strong expression in most of non follicular variant PTC particularly the classic ones.
The specificity of TROP-2 for total PTC cases in all studies was $100 \%$ (all non malignant cases were negative for TROP-2) except for one study where the specificity was $89.3 \%$ [25]. This lowered specificity in that study was attributed to TROP2 positivity in 6 out of 56 non malignant cases; two of them were follicular adenoma and the remaining 4 were non neoplastic cases.

Regarding TROP-2 PPV for total PTC cases, it was $100 \%$ in 4 articles and reduced to $84.6 \%$ in one study [25]. This decline in the PPV may be due to TROP-2 false positive results (6 out of 56 non malignant cases were positive).

TROP-2 NPV in total papillary thyroid carcinoma cases varied between $74.1 \%$ [29] to $92.4 \%$ [30] and its NPV in FVPTC varied between 75.9\% [29] to $98 \%$ [25]. The reduced NPV in total PTC to $(74.1 \%)$ as stated by (Murtezaoglu and Gucer, 2017) [29] may be due to large number of false negative cases because the latter study [29] investigated large number of FVPTC (21 out of 42 PTC were TROP-2 negative, of which 19 cases were of FVPTC and two cases were non FVPTC variant PTC).

There was no great variability in TROP-2 diagnostic accuracy regarding total papillary thyroid carcinoma cases among the studied articles as it ranged from $79.4 \%$ [29] to 94.3 [30]. The accuracy declined more when calculated for FVPTC alone as it ranged from $76.3 \%$ [29] to $93.2 \%$ [30]. That decline in the study of (Murtezaoglu and Gucer, 2017) [29]

The sensitivity of TROP-2 in diagnosing thyroid cancers other than PTC as follicular, medullary, poorly differentiated and anaplastic thyroid carcinomas was very low making it a good novel marker in diagnosing papillary thyroid carcinoma in general and non follicular variant particularly.

\section{Conclusion:}

TROP- 2 could be considered as a reliable immunostaining marker in diagnosing papillary thyroid carcinoma especially non follicular variant owing to its high specificity and sensitivity. TROP2 is a specific rather than sensitive as its specificities in 4 studies revealed $100 \%$. Therefor it is expected to be negative in benign and non-neoplastic thyroid lesions reducing the overdiagnosis of PTC and discriminating it from many mimickers.

\section{References}

1- SEER Cancer Statistics Review (CSR) 1975-2014 [Internet]. Available from, https://seer.cancer. gov/statfacts . /html/thyro.html.. 2017. 
2- HELAL T., SALMAN M. and EZZ-ELARAB S.: Pathology based cancer registry 2001-2010. Ain Shams University Chapter, (5): 89-90, 2015.

3- CRILE JR G. and HAZARD J.B.: Relationship of the age of the patient to the natural history and prognosis of carcinoma of the thyroid. Annals of Surgery, 138 (1): 33, 1953.

4- EL-DEMELLAWY D., NASR A.L., BABAY S. and ALOWAMI S.: Diagnostic utility of CD56 immunohistochemistry in papillary carcinoma of the thyroid. Pathology-Research and Practice, 205 (5): 303-9, 2009.

5- HEITZ P., KOMMINOTH P., PERREN A., KLIMSTRA D., DAYAL Y., BORDI C., et al.: WHO classification of tumours, Pathology and genetics of tumours of endocrine organs. Tumours of the Endocrine Pancreas, 2004.

6- CHEUNG C.C., EZZAT S., FREEMAN J.L., ROSEN I.B. and ASA S.L.: Immunohistochemical diagnosis of papillary thyroid carcinoma. Modern Pathology, 14 (4): 338-42, 2001.

7- PRASAD M.L., PELLEGATA N.S., HUANG Y., NAGARAJA H.N., DE LA CHAPELLE A. and KLOOS R.T. Galectin-3, fibronectin-1, CITED-1, HBME1 and cytokeratin-19 immunohistochemistry is useful for the differential diagnosis of thyroid tumors. Modern Pathology, 18 (1): 48-57, 2005.

8- EL-DEMELLAWY D., NASR A. and ALOWAMI S.: Application of CD56, P63 and CK19 immunohistochemistry in the diagnosis of papillary carcinoma of the thyroid. Diagn. Pathol., 3 (5), 2008.

9- BALOCH Z.W., LIVOLSI V.A., ASA S.L., ROSAI J., MERINO M.J., RANDOLPH G., et al.: Diagnostic terminology and morphologic criteria for cytologic diagnosis of thyroid lesions: A synopsis of the National Cancer Institute Thyroid Fine-Needle Aspiration State of the Science Conference. Diagnostic Cytopathology, 36 (6): 425-37, 2008.

10- SCHMID K.W. and FARID N.R.: How to define follicular thyroid carcinoma? Virchows Archiv., 448 (4): 385-93, 2006.

11- MA H., XU S., YAN J., ZHANG C., QIN S, WANG X, et al.: The value of tumor markers in the diagnosis of papillary thyroid carcinoma alone and in combination. Polish journal of pathology: Official journal of the Polish Society of Pathologists, 65 (3): 202-9, 2014.

12- CUBAS R., LI M., CHEN C. and YAO Q.: TROP2: A possible therapeutic target for late stage epithelial carcinomas. Biochimica et Biophysica Acta (BBA)-Reviews on Cancer, 1796 (2): 309-14, 2009.

13- WENQI D., LI W., SHANSHAN C., BEI C., YAFEI Z., FEIHU B., et al.: EpCAM is overexpressed in gastric cancer and its downregulation suppresses proliferation of gastric cancer. Journal of Cancer Research and Clinical Oncology, 135 (9): 1277-85, 2009.

14- LIPINSKI M., PARKS D.R., ROUSE R.V. and HERZENBERG L.A.: Human trophoblast cell-surface antigens defined by monoclonal antibodies. Proceedings of the National Academy of Sciences, 78 (8): 5147-50, 1981.

15- OHMACHI T., TANAKA F., MIMORI K., INOUE H., YANAGA K. and MORI M.: Clinical significance of
TROP2 expression in colorectal cancer. Clinical Cancer Research, 12 (10): 3057-63, 2006.

16- FONG D., SPIZZO G., GOSTNER J.M., GASTL G., MOSER P., KRAMMEL C., et al.: TROP2: A novel prognostic marker in squamous cell carcinoma of the oral cavity. Modern Pathology, 21 (2): 186-91, 2008.

17- ALBERTI S., MIOTTI S., STELLA M., KLEIN C.E., FORNARO M., MENARD S., et al.: Biochemical characterization of Trop-2, a cell surface molecule expressed by human carcinomas: formal proof that the monoclonal antibodies T16 and MOv-16 recognize Trop-2. Hybridoma., 11 (5): 539-45, 1992.

18- FORNARO M., ARCIPRETE R.D., STELLA M., BUCCI C., NUTINI M., CAPRI M.G., et al.: Cloning of the gene encoding TROP-2, a cell-surface glycoprotein expressed by human carcinomas. International Journal of Cancer 62 (5): 610-8, 1995.

19- FANG Y., LU Z., WANG G., PAN Z., ZHOU Z., YUN J., et al.: Elevated expressions of MMP7, TROP2, and survivin are associated with survival, disease recurrence, and liver metastasis of colon cancer. International Journal of Colorectal. Disease, 24 (8): 875-84, 2009.

20- STEPAN L.P., TRUEBLOOD E.S., HALE K., BABCOOK J., BORGES L. and SUTHERLAND C.L.: Expression of Trop2 Cell Surface Glycoprotein in Normal and Tumor Tissues Potential Implications as a Cancer Therapeutic Target. Journal of Histochemistry \& Cytochemistry, 59 (7): 701-10, 2011.

21- MANGINO G., GRAZIA CAPRI M., BARNABA V. and ALBERTI S.: Presentation of native TROP-2 tumor antigens to human cytotoxic $\mathrm{T}$ lymphocytes by engineered antigen-presenting cells. International Journal of Cancer, 101 (4): 353-9, 2002.

22- WANG J., DAY R., DONG Y., WEINTRAUB S.J., MICHEL L.: Identification of Trop-2 as an oncogene and an attractive therapeutic target in colon cancers. Molecular Cancer Therapeutics, 7 (2): 280-5, 2008.

23- LIU H., SHI J. and LIN F.: The Potential Diagnostic Utility of TROP-2 in Thyroid Neoplasms. Applied immunohistochemistry \& molecular morphology: AIMM/official publication of the Society for Applied Immunohistochemistry, 2016.

24- SIMMS A., JACOB R.P., COHEN C. and SIDDIQUI M.T.: TROP-2 expression in papillary thyroid carcinoma. Diagnostic Cytopathology, 44 (1): 26-31, 2016.

25- ADDATI T., ACHILLE G., CENTRONE M., PETRONI S., POPESCU O., RUSSO S., et al.: TROP-2 expression in papillary thyroid cancer: A preliminary cyto-histological study. Cytopathology, 26 (5): 303-11, 2015.

26- KHURANA K.K., BALOCH Z.W. and LIVOLSI V.A.: Aspiration cytology of pediatric solitary papillary hyperplastic thyroid nodule: Potential pitfall. Archives of Pathology \& Laboratory Medicine, 125 (12): 1575-8, 2001.

27- LIVOLSI V.A.: Papillary neoplasms of the thyroid: Pathologic and prognostic features. American Journal of Clinical Pathology, 97 (3): 426-34, 1992.

28- METE O. and ASA S.L.: Pitfalls in the Diagnosis of Follicular Epithelial Proliferations of the Thyroid. Advances in Anatomic Pathology, 19 (6): 363-73, 2012. 
29- MURTEZAOGLU A.R. and GUCER H.: Diagnostic value of TROP-2 expression in papillary thyroid carcinoma and comparison with HBME-1, galectin-3 and cytokeratin 19. Polish Journal of Pathology, 68 (1): 1$10,2017$.
30- BYCHKOV A., SAMPATANUKUL P., SHUANGSHOTI S. and KEELAWAT S.: TROP-2 immunohistochemistry: A highly accurate method in the differential diagnosis of papillary thyroid carcinoma. Pathology, 48 (5): 425-33, 2016.

\section{الصلاحية التشحيصية لجزئ TROP-2

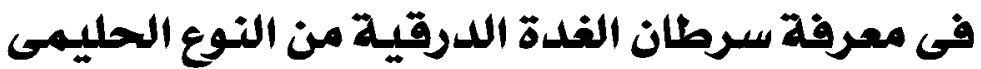

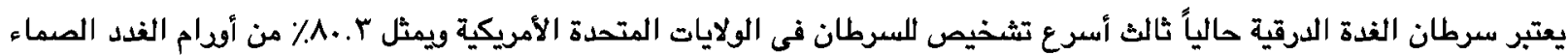

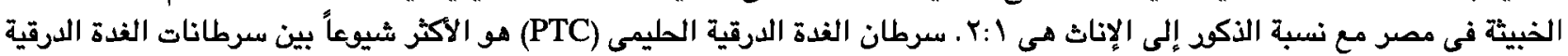

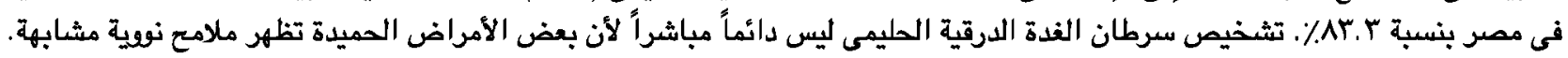

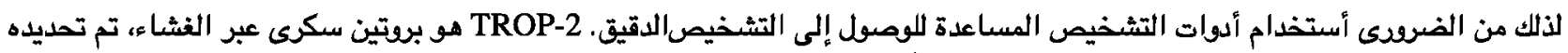

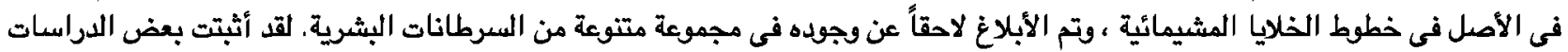
أن الطاقة التشخيصية لـ فROP-2 فى سرطان الغدة الدرقية الحليمى يكمن أن يكن وسيلة محتملة مبتكرة للتشخيص.

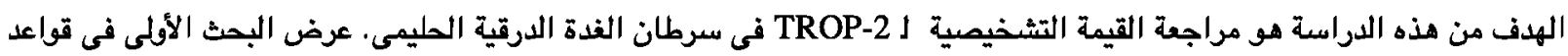

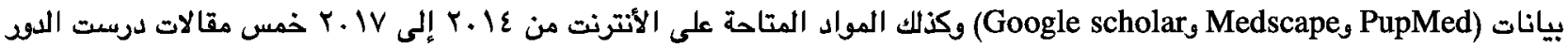
التخفيصى لـ TROP-2 في تمييز سرطان الغدة الدرقية الحليمى عن الآفات غير الخبيثة والتى تم إجرائها على على على عينات نسيجية فقط.

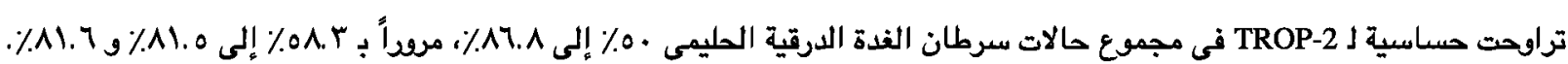

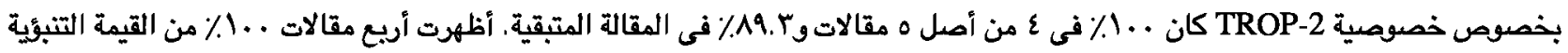

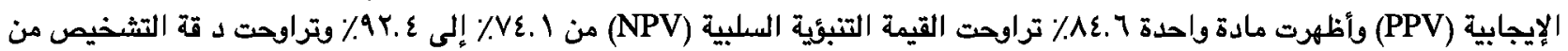

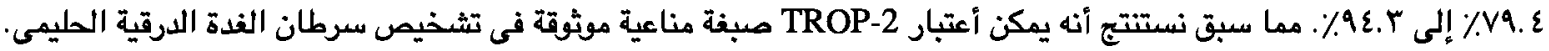

